\title{
Microorganismos eficientes y vermicompost lixiviado aumentan la producción de pepino
}

\section{Efficient microorganism and lixiviate vermicompost applications increase the cucumber production}

\author{
Alexander Calero Hurtado'; Elieni Quintero Rodríguez²; Yanery Pérez Díaz; \\ Yainier González-Pardo Hurtado ${ }^{4}$; Tomasa Norma González Lorenzo ${ }^{5}$
}

1Ing. Agrónomo, M.Sc. Agricultura Sostenible, Estudiante Ph.D. Universidade Estadual Paulista "Júlio de Mesquita Filho" (UNESP). Campus Jaboticabal, São Paulo, Brasil; e-mail: alexcalero34@gmail.com; (Dhttp://orcid.org/0000-0001-6536-2908

²Ing. Agrónoma, Investigadora. Empresa Agropecuaria Agroindustrial "Melanio Hernández". Tuínucu. Taguasco, Sancti Spíritus, Cuba; e-mail: elieni. quintero@gmail.com; Dhttp://orcid.org/0000-0003-4994-9586

${ }^{3}$ Lic. Profesora. Centro Universitario Municipal de Taguasco "Enrique José Varona" (CUM). Universidad de Sancti Spíritus "José Martí Pérez" (UNISS). Zaza de Medio, Taguasco, Sancti Spíritus, Cuba; e-mail: yanery@uniss.edu.cu; Dhttp://orcid.org/0000-0003-4568-1981

${ }^{4}$ Estudiante pregrado. Universidad de Sancti Spíritus "José Martí Pérez" (UNISS), Facultad de Ciencias Agropecuarias. Sancti Spíritus, Cuba; e-mail: yainier@ uniss.edu.cu; Dhttps://orcid.org/0000-0001-7811-353X

${ }^{5}$ Lic. Profesora. Centro Universitario Municipal de Taguasco "Enrique José Varona" (CUM). Universidad de Sancti Spíritus "José Martí Pérez" (UNISS). Zaza de Medio, Taguasco, Sancti Spíritus, Cuba; e-mail: normag@uniss.edu.cu; Dhttps://orcid.org/0000-0003-4887-1667

Cómo citar: Calero Hurtado, A.; Quintero Rodríguez, E.; Pérez Díaz, Y.; González-Pardo Hurtado, Y.; González Lorenzo, T.N. 2019. Microorganismos eficientes y vermicompost lixiviado aumentan la producción de pepino Rev. U.D.C.A Act. \& Div. Cient. $22(2): e 1167$. http://doi.org/10.31910/rudca.v22.n2.2019.1167

Artículo de acceso abierto publicado por Revista U.D.C.A Actualidad \& Divulgación Científica, bajo una licencia Creative Commons CC BY-NC 4.0

Recibido: Febrero 11 de 2019

Aceptado: Octubre 29 de 2019

Editado por: Ingeborg Zenner de Polanía

\section{RESUMEN}

Los principales problemas de la producción de pepino, residen mayormente en la escasez de fertilizantes minerales, afectaciones climatológicas y limitado uso de los biofertilizantes, por lo cual, es importante buscar alternativas eficientes que aumenten la productividad, la racionalidad y la sustentabilidad. El objetivo de esta investigación fue evaluar la utilización individualizada y asociada de los biofertilizantes microorganismos eficientes y vermicompost lixiviado en el incremento agroproductivo del pepino. El trabajo fue desarrollado en la unidad productiva "El Estadio", Sancti Spíritus,
Cuba, entre enero a abril de 2015 y fue utilizado el cultivar Su Yi Sung de pepino. Los tratamientos aplicados fueron un control, inoculación al suelo y aplicaciones foliares de Microorganismos eficientes y vermicompost lixiviado, a 100 y $200 \mathrm{~mL} \mathrm{~L}^{-1}$ y la inoculación al suelo, con Microorganismos eficientes, a $100 \mathrm{~mL} \mathrm{~L}^{-1}$ y aplicaciones foliares con vermicompost lixiviado, a $100 \mathrm{~mL} \mathrm{~L}^{-1}$. Las variables evaluadas fueron el número de hojas, de flores femeninas y de frutos por planta, longitud de frutos $(\mathrm{cm})$, masa de los frutos (g) y el rendimiento $\left(\mathrm{kg} \mathrm{m}^{-2}\right)$. Los resultados mostraron que la aplicación individual y combinada de los biofertilizantes tuvieron un efecto bioestimulante en la producción de pepino y la aplicación 
de microorganismos eficientes a $100 \mathrm{~mL} \mathrm{~L}^{-1}$ y la combinación con vermicompost lixiviado a $100 \mathrm{~mL} \mathrm{~L}^{-1}$ constituyen una alternativa en la productividad del cultivo, especialmente, porque aumentaron el número de hojas, flores femeninas, frutos, masa y longitud de los frutos e incrementaron el rendimiento en $42 \%$ con relación al tratamiento control.

Palabras clave: aplicación foliar; biofertilizantes; Cucumis sativus; inoculación del suelo; rendimiento.

\section{ABSTRACT}

The main problems of cucumber production reside fundamentally by the mineral fertilizer's shortage, climatic affectation and limited use of biofertilizers, which is important to looking for efficient alternatives that increase productivity, rationality and sustainability. The objective of this research was to evaluate the effect of individualized and associated use of biofertilizers efficient microorganisms and lixiviate vermicompost on the agro-productive increase of cucumber. The work was developed in the productive unit "El Estadio", Sancti Spíritus, Cuba, between January to April 2015 and Su Yi Sung cultivar of cucumber was used. The treatments used were a control (no application), soil inoculation and foliar applications with efficient microorganisms and lixiviate vermicompost at 100 and $200 \mathrm{~mL} \mathrm{~L}^{-1}$, and soil inoculation with efficient microorganisms at $100 \mathrm{~mL} \mathrm{~L}^{-1}$ and foliar applications with lixiviate vermicompost at $100 \mathrm{~mL} \mathrm{~L}^{-1}$. The following variables were observed: number of leaves, female flowers and fruits per plant, length of fruits $(\mathrm{cm})$, mass of fruits (g) and yield $\left(\mathrm{kg} \mathrm{m}^{-2}\right)$. The results showed that the individual and combined application of biofertilizers had a biostimulants effect on cucumber production. The efficient microorganism's application at $100 \mathrm{~mL} \mathrm{~L}^{-1}$ and the combination with lixiviate vermicompost to $100 \mathrm{~mL} \mathrm{~L} \mathrm{~L}^{-1}$ constituting a sustainable alternative in the cucumber productivity, especially because they increased the number of leaves, female flowers, fruits, mass and length of fruits and increasing the yield by $42 \%$ compared to control treatment.

Keywords: biofertilizer; foliar application; Cucumis sativus; soil inoculation; yield.

\section{INTRODUCCIÓN}

El pepino Cucumis sativus (L.) es una hortaliza que se produce en varios países del mundo y puede ser cultivado en condiciones de campo o en invernaderos, en dependencia de las condiciones climatológicas (Mohammadi \& Omid, 2010).

En Cuba, el C. sativus es cultivado en condiciones de campo, casas de cultivo y más recientemente en la Agricultura Urbana y Suburbana. Un rasgo característico de la producción de pepino, se debe, tanto a la variación del área cultivada como a los rendimientos, que se ven muy afectados por las condiciones climáticas desfavorables que retrasan las fechas agrotécnicas y las fases fenológicas (Gómez et al. 1986; Cabrera et al. 2007).
El empleo de los microorganismos, como biofertilizantes para especies vegetales cultivadas, ha sido una práctica común en los últimos años. Las rizobacterias promotoras del crecimiento de las plantas (RPCP) se han destacado como biofertilizantes, porque estos microorganismos se adaptan y crecen rápidamente alrededor de las raíces de las plantas (Ahirwar et al. 2015; Kumar \& Saraf, 2015; Rashid et al. 2016).

Los microorganismos eficientes (ME) es una tecnología desarrollada por el profesor Teuro Higa, basada en una mezcla microbiana de diferentes géneros de microrganismos (bacterias, hongos, levaduras, entre otros). Esta tecnología es actualmente aplicada en diversos proceso agropecuarios y medioambientales y empleada en más de 80 países (Arias Hoyos, 2010). El principio fundamental de esta tecnología consiste en la introducción de un conjunto de microorganismos benéficos, que permiten mejorar las propiedades del suelo (Pedraza et al. 2010). La utilización de los ME ha resultado favorable para la agricultura, porque su introducción a favorecido y beneficiado a los agricultores (Luna \& Mesa, 2016) y la producción de algunos cultivos, como la col (Álvarez et al. 2012), el tomate (Olivera et al. 2015), la zanahoria (Núñez et al. 2017), la cebolla (Liriano et al. 2015) y el frijol (Calero et al. 2016; Calero et al. 2017; Calero et al. 2018; Quintero et al. 2018) y todavía no han sido reportados resultados de su utilización en el cultivo del pepino.

El proceso de vermicompostaje produce lixiviados, debido a las actividades de los microorganismos y el drenaje de los lixiviados es importante para evitar la saturación del producto. Por lo tanto, los lixiviados derivados del vermicompost o vermicompost lixiviado (VL), se consideran beneficiosos y se pueden usar como fertilizantes líquidos, debido a la alta concentración de nutrientes de las plantas (Jarecki et al. 2005; Gutiérrez et al. 2008; Tejada et al. 2008).

Por otro lado, para aumentar la productividad de los cultivos existen algunos productos orgánicos líquidos, como el vermicompost lixiviado (Pant et al. 2009; Preciado et al. 2011). Del mismo modo, Gutiérrez et al. (2008) y Tejada et al. (2008) informaron que los lixiviados de vermicompost son beneficiosos para la producción y la calidad de las cosechas y recomendaron que estos se puedan utilizar para fines agrícolas.

Si es conocido que la utilización de los bioproductos desde el establecimiento inicial del cultivo son esenciales para el éxito de la producción es pertinente evaluar las siguientes hipótesis: la aplicación individual de los bioproductos microorganismos eficientes y vermicompost lixiviado incrementan los indicadores morfométricos y productivos del pepino, con el consecuente aumento de la productividad y, aún, es posible maximizar la respuesta agroproductiva del cultivo, con la aplicación combinada de ambos bioproductos y, cuál de ellos, podría ser más promisorio para la producción de pepino en condiciones de organoponía. Para comprobar estas hipótesis, el objetivo de esta investigación fue evaluar el efecto de la utilización individualizada y asociada entre ME y VL en el incremento agroproductivo del pepino en condiciones de organopónico. 


\section{MATERIALES Y MÉTODOS}

Condiciones de cultivo y material vegetal. El trabajo, se realizó en la unidad productiva "El Estadio", perteneciente a la Agricultura Urbana y Suburbana de la provincia de Sancti Spíritus, Cuba, entre enero a abril de 2015. Las variables climáticas fueron registradas por la Estación Provincial de Sancti Spíritus, temperatura media diaria fue de $22,52^{\circ} \mathrm{C}$, humedad relativa media diaria $78,25 \%$ y precipitación pluvial acumulada de $117,25 \mathrm{~mm}$.

Fue utilizada la variedad Su Yi Sung de pepino (Cucumis sativus L.), obtenida en la Empresa Provincial de Semillas de Sancti Spíritus, con un $97 \%$ de germinación; este cultivar, se puede sembrar todo el año, debido a su rusticidad, porque las plantas crecen vigorosamente, las hojas son de color verde oscuro, los frutos son rectos y alargados, de color verde oscuro, con diámetro entre 5 y $6 \mathrm{~cm}$, una longitud entre 30 a $50 \mathrm{~cm}$ y una masa entre 900 y $950 \mathrm{~g}$. La siembra fue realizada de forma manual a la distancia de $0,90 \mathrm{~m}$ entre líneas y $0,25 \mathrm{~m}$ entre plantas. Dos semillas por hoyo fueron depositadas en los canteros y a los 10 días posteriores a la emergencia (dpe) fue realizado un raleo, para dejar una planta por nido. El cantero recibió de base la aplicación de abono orgánico (compost), a razón de $0,5 \mathrm{~kg} \mathrm{~m}^{-2}$.

Diseño experimental y tratamientos. El diseño experimental utilizado fue bloques al azar, con seis tratamientos y cinco réplicas, para formar 30 parcelas de $3,0 \mathrm{~m}^{2}$. Fueron muestreadas 50 plantas por tratamientos, en el área útil $\left(1,15 \mathrm{~m}^{2}\right)$ y se aplicaron las siguientes variantes: Control (sin aplicación), inoculación al suelo y aplicaciones foliares de ME a $100 \mathrm{~mL} \mathrm{~L}^{-1}$ (ME100), inoculación al suelo y aplicaciones foliares de ME a 200 $\mathrm{mL} \mathrm{L}^{-1}$ (ME200), inoculación al suelo y aplicaciones foliares de Vermicompost lixiviado a $100 \mathrm{~mL}$ $\mathrm{L}^{-1}$ (VL100), inoculación al suelo y aplicaciones foliares de VL a $200 \mathrm{~mL} \mathrm{~L}^{-1}$ (VL200) y la inoculación al suelo con ME a $100 \mathrm{~mL} \mathrm{~L}^{-1}$ y aplicaciones foliares de VL a $100 \mathrm{~mL} \mathrm{~L}^{-1}$ [ME+VL (100)]. Las concentraciones utilizadas del bioproducto ME, se basaron según los resultados alcanzados por Calero et al. (2018) y la VL era las que utilizaban en la entidad.

La inoculación al suelo con los bioproductos ME y VL fue efectuada en el momento de la siembra y las aplicaciones foliares fueron realizadas a los 15 y 30ddg, con apoyo de una asperjadora manual (ECHO MS-21H), de 7,6L de capacidad.

Características de los bioproductos. El inóculo de microorganismos eficientes fue adquirido en la Sucursal de Labiofam de Sancti Spíritus", compuesto por Bacillus subtilis B/23-45-10 Nato $\left(5,4 \times 10^{4} \mathrm{UCFmL}^{-1}\right)$, Lactobacillus bulgaricum B/103-4-1 (3,6×10 $\left.\mathrm{UCFmL}^{-1}\right)$, y Saccharomyces cereviciae L-25-7-12 (22,3×10 $\left.\mathrm{UCFmL}^{-1}\right)$, con certificado de calidad, emitido por el Instituto Cubano de Investigaciones de los Derivados de la Caña de Azúcar (ICIDCA), código R-ID-B-Prot-01-01, según la metodología propuesta por Olivera et al. (2014) y caracterizado por López et al. (2017), con las siguientes características: materia seca: $17,0 \mathrm{gL}^{-1}$; materia orgánica: 1100,0 $\mathrm{mg} \mathrm{L}^{-1}$; $\mathrm{pH}: 3,16$; nitrógeno amoniacal $\left(\mathrm{N}_{-} \mathrm{NH}_{4}{ }^{+}\right): 0,03 \mathrm{mg}$ $\mathrm{L}^{-1}$; potasio $\left(\mathrm{K}^{+}\right): 0,80 \mathrm{mg} \mathrm{L}^{-1}$; fósforo $\left(\mathrm{H}_{2} \mathrm{PO}_{4}\right)$ : $0,83 \mathrm{mg} \mathrm{L}^{-1}$; calcio $\left(\mathrm{Ca}^{2+}\right): 24,05 \mathrm{mg} \mathrm{L}^{-1}$ y magnesio $\left(\mathrm{Mg}^{2+}\right): 4,86 \mathrm{mg} \mathrm{L}^{-1}$. El vermicompost lixiviado se recolectó en la misma entidad, a partir de producción de humus. Los desechos vegetales fueron recogidos y se cortaron finamente en trozos de $5 \mathrm{~cm}$ y se colocaron en predescomposición aeróbicamente durante 15 días en el vivero cementado de $300 \mathrm{dm}^{3}$; posteriormente, fue mezclado con torta de filtro a 1:1 relación en una base de peso. Luego, se introdujeron individualmente 500 ejemplares de Eisenia foetida adultas en 40kg en los residuos biológicos, previamente descompuestos. Para mantener la humedad los riegos fueron por aspersión en un rango de tres días, aproximadamente.

Variables evaluadas. Número de hojas $(\mathrm{NH})$, de flores femeninas $(\mathrm{NF})$, de frutos por planta (FP), longitud de los frutos (LF) (cm), masa de los frutos por planta $(\mathrm{MF})(\mathrm{g})$ y el rendimiento $\left(\mathrm{kg} \mathrm{m}^{-2}\right)$.

Análisis estadísticos. Los datos obtenidos fueron sometidos a un test de Shapiro-Wilk, para determinar la distribución normal; comprobada la normalidad, fue realizada la homogeneidad de la varianza, a través de la prueba de Levene. Una vez comprobada la normalidad y la homogeneidad de los datos, se realizó un análisis de varianza (ANOVA) y cuando fue significativo al 5\% de probabilidad de error, las medias fueron contrastadas mediante la prueba de Rangos Múltiples de Tukey $(\mathrm{P}<0,05)$. Los datos fueron procesados en el software $\mathrm{R}$ ( $\mathrm{R}$ team core, 2018).

\section{RESULTADOS Y DISCUSIÓN}

El número de hojas por planta $(\mathrm{NH})$ fue significativamente $(\mathrm{P}<$ $0,05)$ superior con la aplicación de ME100 individual, sin diferencias estadísticas a la aplicación individual de VL100, pero sí mostró una alta significancia en relación con las variantes ME200, VL200, $\mathrm{ME}+\mathrm{VL}$ (100) y el tratamiento control (Figura 1). Este aumento del $52 \%$ el $\mathrm{NH}$ en relación con el tratamiento control alcanzado por la aplicación del bioproducto ME, se pudo deber a que los microorganismos del suelo desempeñan un papel importante en diferentes transformaciones químicas en los suelos, que influyen en la disponibilidad de macro y micronutrientes para las plantas (Ravindran et al. 2016). También pueden sintetizar y producir fitohormonas, tales como auxinas, citoquininas, giberelinas, el etileno, que logran afectar la proliferación celular en la arquitectura de la raíz, con un aumento de la absorción de nutrientes y de agua (Simranjit et al. 2019). Estos efectos benéficos de la aplicación de los ME en el mejoramiento de la arquitectura de las plantas fueron reportados anteriormente en diferentes especies de plantas, como la fresa (Álvarez et al. 2018), el frijol (Calero et al. 2019a) y el tabaco (Calero et al. 2019b).

Se observa también un efecto benéfico en el aumento del NH con la aplicación foliar del VL comparado con el control (Figura 1). Este aumento del NH con la utilización del VL fue reportado en el cultivo del rábano, por Fleitas et al. (2013) y en otras especies, como sorgo y tomate (Gutiérrez et al. 2008; Jarecki et al. 2005; Tejada et al. 2008).

La producción del NF fue significativamente $(\mathrm{P}<0,05)$ mayor en los tratamientos con ME100 y ME+VL (100), en relación con la utilización de ME200, VL200 y el control (Figura 2). 


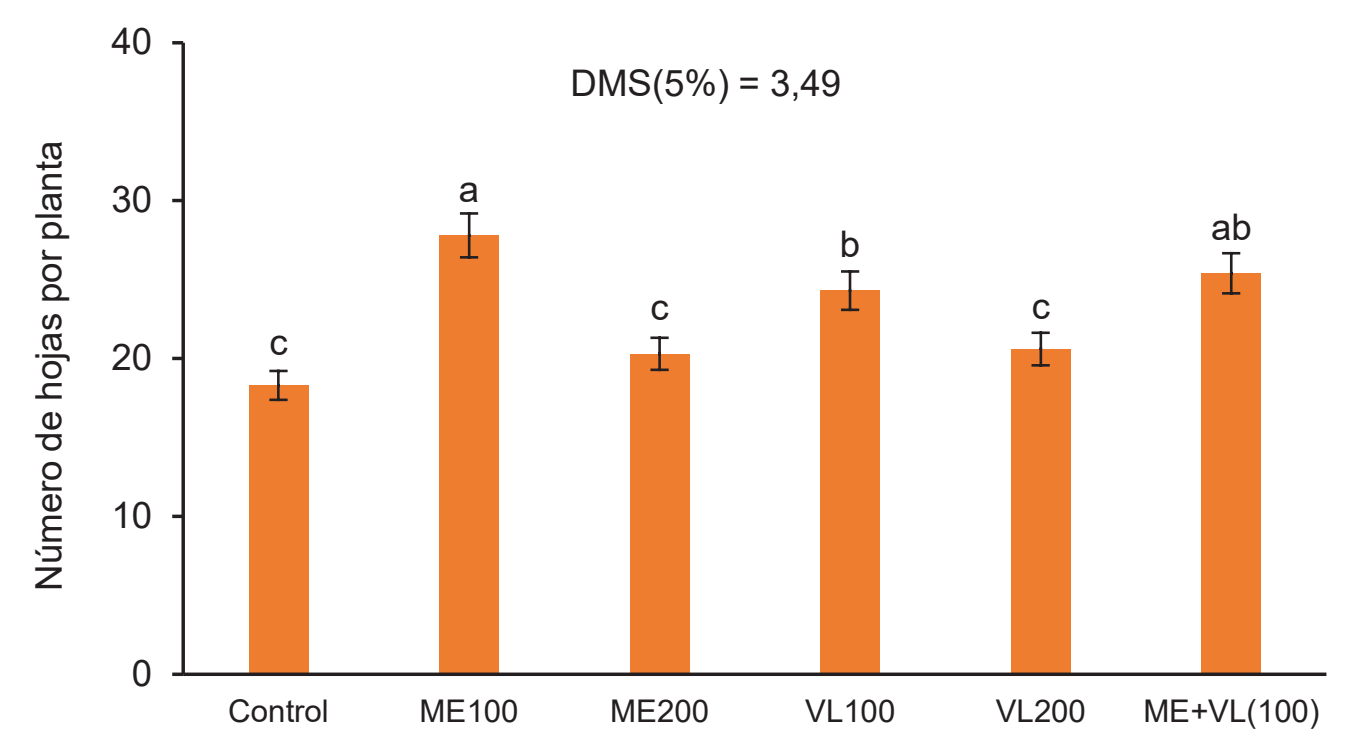

Figura 1. Efecto de los bioproductos en el número de hojas por planta de pepino en el cv. Su Yi Sung. Letras diferentes difieren según Tukey $(\mathrm{p}<0,05)$. Valores representados por las medias \pm error estándar $(\mathrm{EE})$ de cinco repeticiones $(\mathrm{Medias} \pm \mathrm{EE}, \mathrm{n}=5)$. CV $(\%)=$ 7,$95 ; \mathrm{DE}( \pm)=1,76$.

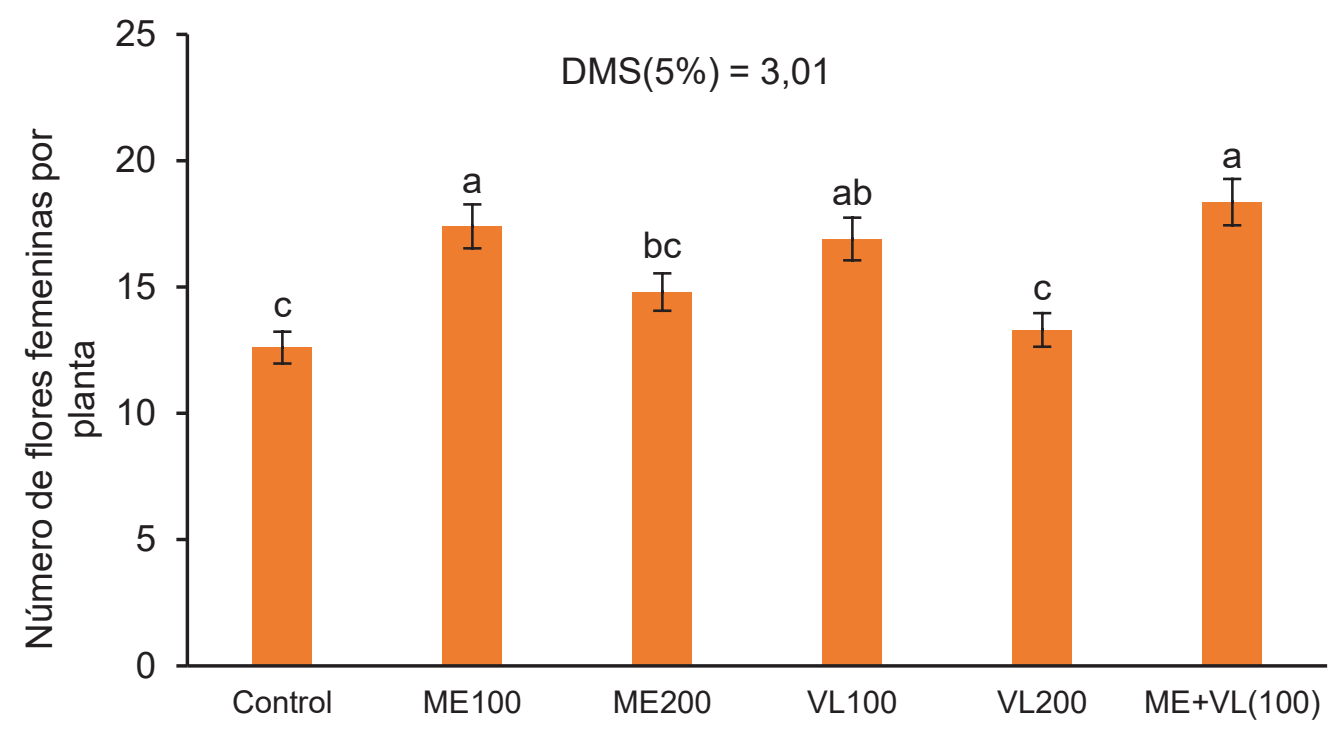

Figura 2. Efecto de los bioproductos en el promedio de flores femeninas por planta de pepino en el cv. Su Yi Sung. Letras diferentes difieren según Tukey $(\mathrm{p}<0,05)$. Medias \pm EE, $\mathrm{n}=5$. CV $(\%)=9,58 ; \mathrm{DE}( \pm)=1,52$.

La aplicación de $\mathrm{ME}$ a $100 \mathrm{~mL} \mathrm{~L}^{-1}$ tuvo un efecto benéfico en el aumento del NF en plantas de pepino, conjuntamente con la aplicación foliar de VL100 y la combinación entre ME+VL (100), comparada con los demás bioproductos y propiciaron un incremento de $39 \%$ en relación con el control. Este aumento pudo estar relacionado con el incremento en el NH (Figura 1), influenciado por una mejora en la arquitectura de las plantas. Por tanto, este incremento en el NF en este cultivo, con el empleo de los ME y el VL, pudo estar ocasionado por la incorporación de sustancias y de elementos que estimulan el crecimiento de las plantas, presentes en su composición (López et al. 2017; Gutiérrez et al. 2008; Jarecki et al. 2005; Tejada et al. 2008), hecho también observado por Olivera et al. (2015), en plantas de tomate y en frijol (Calero et al. 2018; Quintero et al. 2018). 
El FP fue estadísticamente $(\mathrm{P}<0,05)$ superior cuando se aplicaron los bioproductos ME100, VL100 y la combinación entre ellos ME+VL (100), en relación con la utilización de los tratamientos con ME200, VL200 y el control (Figura 3). La aplicación de estos bioproductos incrementó en $63 \%$ el FP en relación con los tratamientos con ME200, VL200 y el control. Este resultado benéfico fue obtenido con la aplicación foliar de ME100, VL100 y la combinación de ME+VL (100), por el hecho de que aumentaron el NH (Figura 1) y el NF (Figura 2), con el consecuente incremento de la LF, la MF y la productividad de los frutos (Figuras 4,5,6).

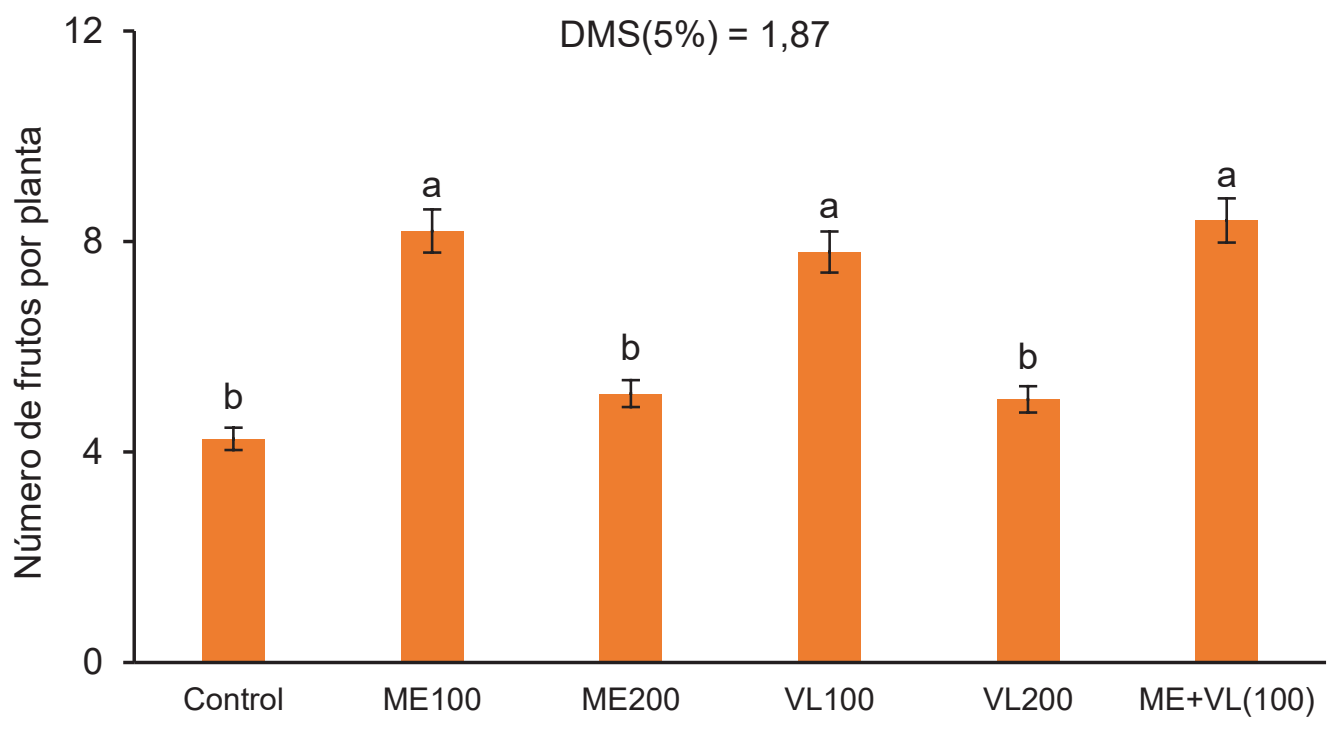

Figura 3. Efecto de los bioproductos en el número de frutos por planta de pepino en el cv. Su Yi Sung. Letras diferentes difieren según Tukey $(\mathrm{p}<0,05)$. Medias \pm EE, $\mathrm{n}=5$; CV $(\%)=14,95 ; \mathrm{DE}( \pm)=0,94$.

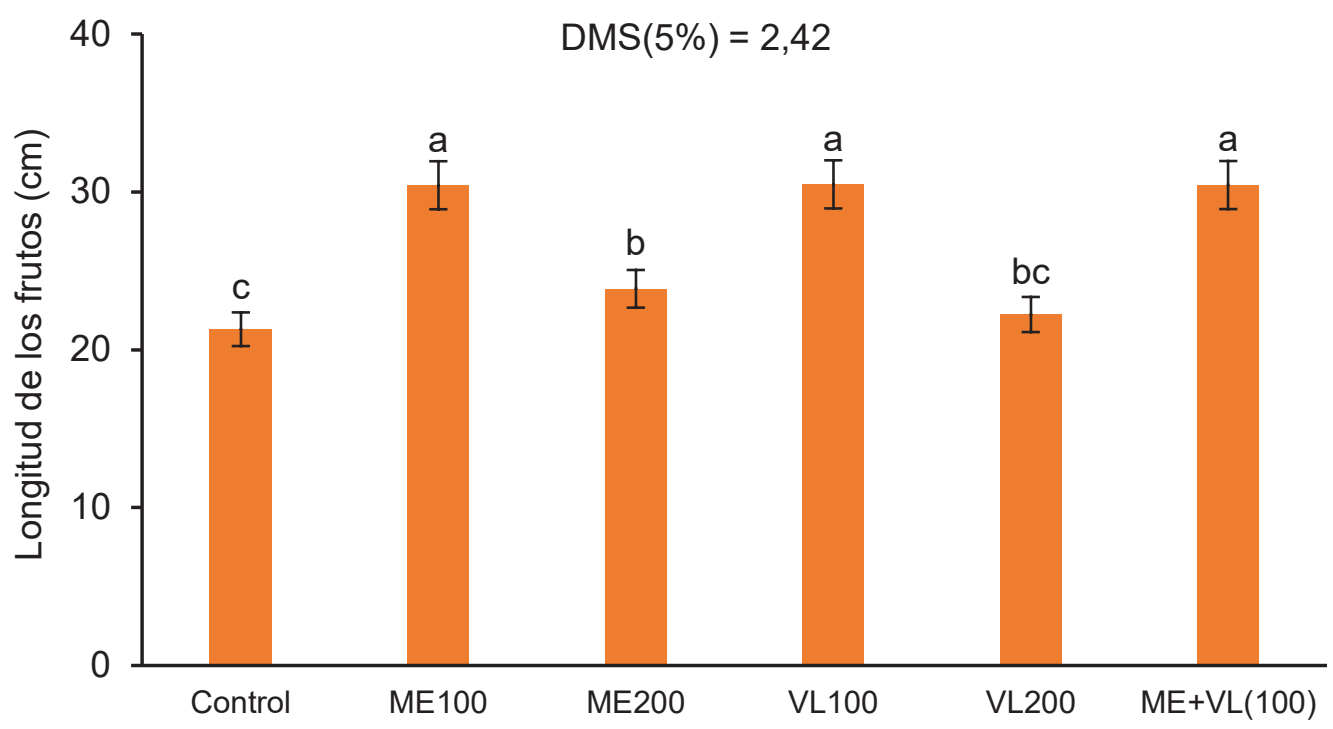

Figura 4. Efecto de los bioproductos en la longitud de los frutos de pepino en el cv. Su Yi Sung. Letras diferentes difieren según Tukey $(\mathrm{p}<0,05)$. Medias $\pm \mathrm{EE}, \mathrm{n}=5 ; \mathrm{CV}(\%)=4,64 ; \mathrm{DE}( \pm)=1,22$. 


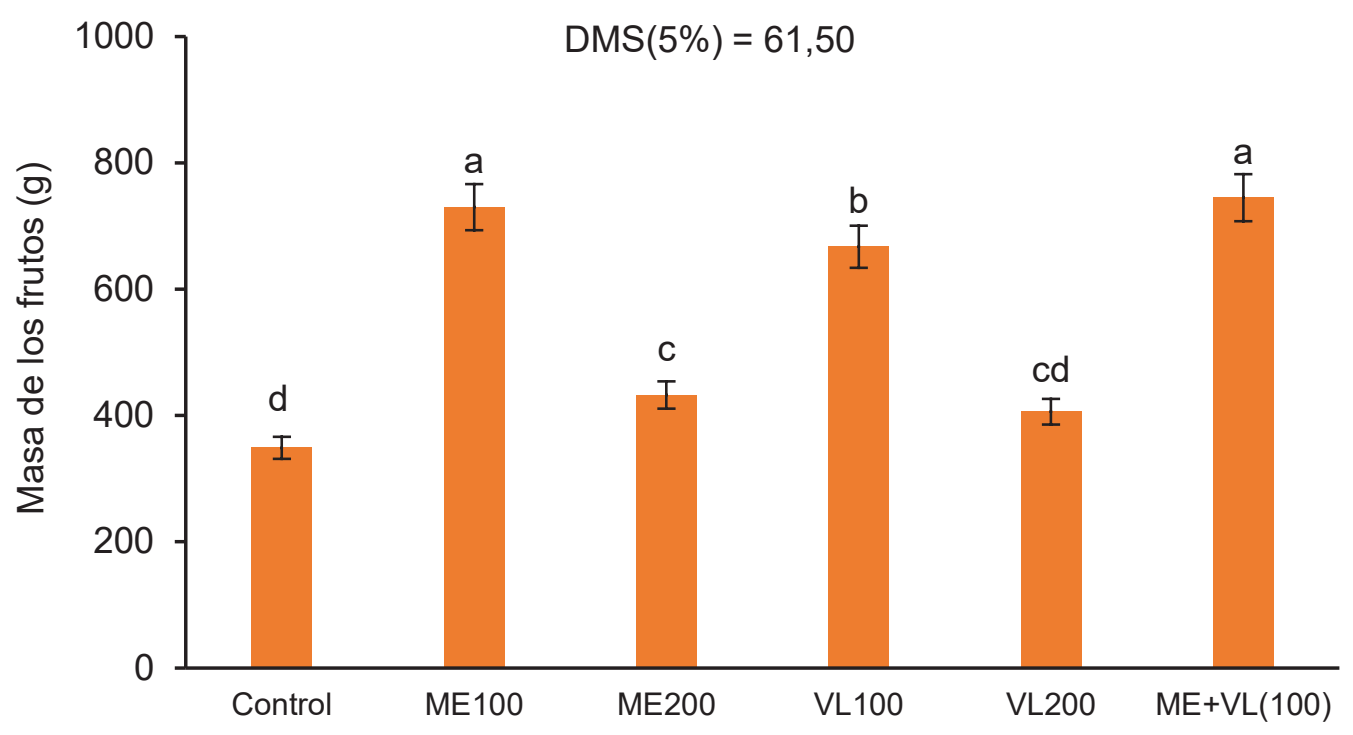

Figura 5. Efecto de los bioproductos en la masa promedio del fruto de pepino en el cv. Su Yi Sung. Letras diferentes difieren según Tukey $(\mathrm{p}<0,05)$. Medias $\pm \mathrm{EE}, \mathrm{n}=5 ; \mathrm{CV}(\%)=5,58 ; \mathrm{DE}( \pm)=30,93$.

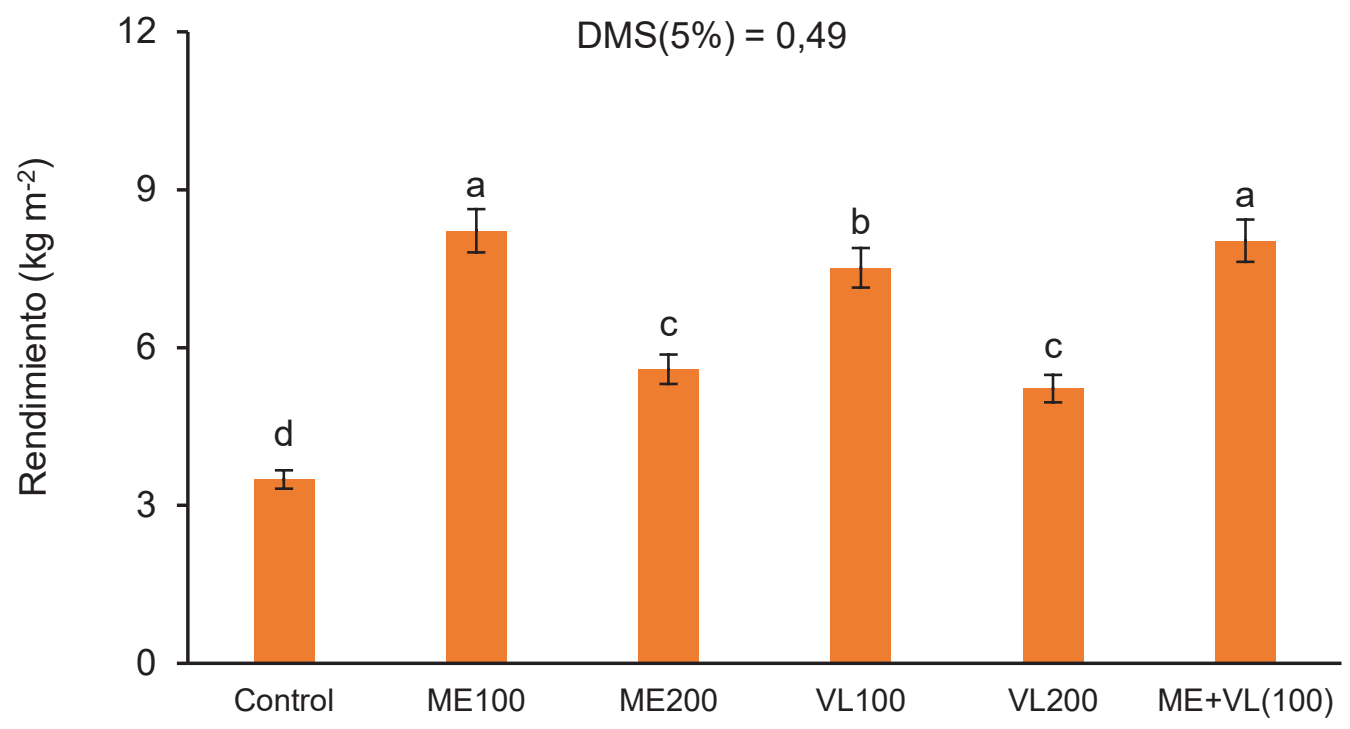

Figura 6. Efecto de los bioproductos en el rendimiento $\left(\mathrm{kg} \mathrm{m}^{-2}\right)$ del pepino en el cv. Su Yi Sung. Letras diferentes difieren según Tukey ( $\mathrm{p}$ $<0,05)$. Medias \pm EE, $\mathrm{n}=5 ; \mathrm{CV}(\%)=3,85 ; \mathrm{DE}( \pm)=0,25$.

En las plantas de pepino quedó evidenciado que la utilización del bioproducto ME aumentó del FP. Estos efectos positivos en el incremento del número de frutos con la aplicación de los ME fueron reportados anteriormente en plantas de tomate (Olivera et al. 2015), de zanahoria (Núñez et al. 2017) y de frijol (Calero et al. 2018), mientras que la aplicación del VL fue benéfico en el aumento del FP, hecho observado por Fleitas et al. (2013), en plantas de rábano.

La longitud de los frutos fue superior con la aplicación de los bioproductos a las concentraciones menores ME100, VL100 y la combinación entre ellos ME+VL (100), comparados con las mayores concentraciones de ME200, VL200 y el tratamiento control (Figura 4).

Este aumento del $43 \%$ alcanzado en la longitud de los frutos, por la aplicación de los bioproductos ME100, VL100 y la combinación de ellos en relación con el control, se pudo deber al incremento del NH, NF y FP (Figuras 1, 2,3), lo que provocó un incremento en el tamaño de los frutos (Figura 4). Estos resultados benéficos logrados por la inoculación y aplicación foliar de los ME, en el aumento del 
tamaño y volumen de los frutos, fueron comprobados anteriormente en plantas de pepino (Zhao et al. 2017), de fresa (Álvarez et al. 2018) y de frijol (Calero et al. 2019a; c).

$\mathrm{Al}$ respecto, Banerjee et al. (2010) plantearon que la inoculación de suelos o semillas con ME mejoran el rendimiento de las cosechas, porque aumentan la disponibilidad de fósforo y otras sustancias estimulantes. Singh et al. (2011) indicaron que la inoculación de grupos microbianos favorece el crecimiento de las plantas, porque envuelven diferentes procesos, como la fijación de nitrógeno atmosférico, la disponibilidad de nutrientes esenciales y la promoción del desarrollo de las plantas, además de incrementar la productividad y la calidad de las cosechas.

La aplicación de la menor concentración del VL100 incrementó el LF del pepino comparado con el control (Figura 4). Este resultado, se podría deber a la composición microbiana del bioproducto, que favorecieron el crecimiento de las plantas de pepino; todos los consorcios microbianos no muestran el mismo efecto. Los microorganismos presentan múltiples beneficios para las plantas, la interacción microbiana en el suelo es bastante amplia y los beneficios fueron evidentes. Estos efectos fueron demostrados anteriormente en plantas de pepino (Zhao et al. 2017) y otras especies de plantas, como la fresa (Álvarez et al. 2018) y el frijol (Calero et al. 2019a).

La masa promedio de los frutos $(\mathrm{MF})$ fue similar en los tratamientos con ME100 y ME+VL (100) con incrementos significativos, en relación con la aplicación de VL100 individual y los tratamientos ME200, VL200 y el control (Figura 5).

La aplicación foliar de los bioproductos ME100 y la combinación entre ME+VL (100) tuvieron un efecto bioestimulante comparado con las demás variantes utilizadas y aumentaron en 114\% la MF en relación con el control, porque aumentaron el NH, NF, FP y la LF, lo que significó un incremento en la masa de los frutos. Estos resultados benéficos de la aplicación del bioproducto ME en el incremento de la masa de los frutos fueron reportados anteriormente en diferentes especies de plantas, como la zanahoria (Núñez et al. 2017) y el frijol (Calero et al. 2016; 2017; 2018; 2019a; Quintero et al. 2018).

Los rendimientos obtenidos demuestran que los tratamientos con ME100 y la combinación entre ME+VL (100) causaron un efecto estimulante significativamente superior comparado con la aplicación individual de VL100 y los tratamientos ME200, VL200 y el control (Figura 6), probablemente, porque fue evidenciado una mejora en los indicadores morfométricos NH y NF (Figuras 1 y 2) y de los parámetros productivos NF, LF y MF (Figuras 3, 4 y 5). Por otra parte, los microorganismos aplicados en los diferentes bioproductos favorecieron el crecimiento de las plantas de pepino, demostrándose que todos los consorcios microbianos no muestran el mismo efecto. Este efecto positivo de la adición de los ME fue observado anteriormente en plantas de fresa (Álvarez et al. 2018) y de frijol (Calero et al. 2019c).
Los efectos benéficos de la aplicación de los bioproductos ME100 y la combinación entre ME+VL (100) favorecieron el rendimiento del pepino, porque incrementaron en $13 \%$ el rendimiento en relación con el tratamiento control. Este aumento del rendimiento es posible, porque el empleo de los microorganismos promotores del crecimiento produce diversas sustancias, como hormonas y nutrientes, que favorecen la productividad de los cultivos (Rashid et al. 2016; Zahedi, 2016).

De acuerdo con Calero et al. (2016), la aplicación foliar de ME mezclado con bioestimulantes aumentó el rendimiento en $79 \%$ en relación con la variante sin aplicación. Estos efectos positivos de los ME en el incremento del rendimiento fueron demostrados para otras hortalizas, como col, cebolla y zanahoria (Álvarez et al. 2012; Liriano et al. 2015; Núñez et al. 2017). Por otro lado, el efecto de la utilización del vermicompost lixiviado $\left(100 \mathrm{~mL} \mathrm{~L}^{-1}\right)$ incrementó el rendimiento del pepino en 115\% comparado con el control; este efecto bioestimulante fue demostrado en plantas de maíz, por Preciado et al. (2014) y en la fresa (Singh et al. 2010).

Finalmente, la hipótesis estudiada fue verificada, indicando que, el efecto bioestimulante de los bioproductos fue demostrada con la inoculación al suelo y aplicación foliar de ME, a la concentración de $100 \mathrm{~mL} \mathrm{~L}^{-1}$, hechos no observado en los demás tratamientos en la producción de pepino. Por tanto, la aplicación de bioproducto microorganismos eficientes fue más promisorio en la productividad del pepino que vermicompost lixiviado y ambos constituyen una alternativa sostenible en la productividad del cv. Su Yi Sung de pepino, en condiciones de organoponía.

Agradecimientos: Los autores agradecen a la Sucursal de Labiofam, por ofrecer gratuitamente los bioproductos para el desarrollo de estudio. También agradecen a la Cooperativa de Créditos y Servicios "Mártires de Taguasco", por la disponibilidad de recursos para el acompañamiento del trabajo en campo. Conflicto de intereses: Los autores realizaron aportes significativos al documento y declaran que no está considerado para su publicación en ninguna otra revista y no tener conflicto de intereses y están de acuerdo con su publicación. Financiación: Todos los recursos y gastos de la investigación fueron financiados por la Cooperativa de Créditos y Servicios "Mártires de Taguasco".

\section{REFERENCIAS}

1. AHIRWAR, N.K.; GUPTA, G.; SINGH, V.; RAWLLEY, R.K.; RAMANA, S. 2015. Influence on growth and fruit yield of tomato (Lycopersicon esculentum Mill.) plants by inoculation with Pseudomonas fluorescence (SS5): Possible role of plant growth promotion. Int. J. Curr. Microbiol. App. Sci. (India). 4(2):720-730.

2. ÁLVAREZ, J.; NÚÑEZ, D.; LIRIANO, R.; TERENCE, G. 2012. Evaluación de la aplicación de microorganismos eficientes en col de repollo (Brassica oleracea L.) en condiciones de organopónico semiprotegido. Cent. Agrícola. (Cuba). 39(4):27-30. 
3. ÁlvareZ, M.; TUCTA, F.; QUisPe, E.; MEZA, V. 2018. Incidencia de la inoculación de microorganismos benéficos en el cultivo de fresa (Fragaria sp.). Sci. Agropecu. (Perú). 9(1):33-42. http://dx.doi.org/10.17268/sci. agropecu.2018.01.04

4. ARIAS HOYOS, A. 2010. Microrganismos eficientes y su beneficio para la agricultura y el medio ambiente. J. Cienca e Ingeniería. (Colombia). 02(02):42-45.

5. BANERJEE, S.; PALIT, R.; SENGUPTA, C.; STANDING, D. 2010. Stress induced phosphate solubilization by "Arthrobacter" sp. and "Bacillus" sp. isolated from tomato rhizosphere. Aust. J. Crop Sci. (Australia). 4(6):378-383.

6. CABRERA, A.; ARZUAGA, J:; MOJENA, M. 2007. Desbalance nutrimental del suelo y efecto sobre el rendimiento de tomate (Lycopersicon solanum $\mathrm{L}$.) y pepino (Cucumis sativus L.) en condiciones de cultivo protegido. Cultiv. Trop. (Cuba). 28(3):91-97.

7. CALERO, A.; PÉREZ, Y.; QUINTERO, E.; OLIVERA, D.; PEÑA, K. 2019a. Efecto de la aplicación asociada entre Rhizobium leguminosarum y microorganismos eficientes sobre la producción del fríjol común. Cienc. Tecnol. Agropecu. (Colombia). 20(2):309-322. https://doi.org/10.21930/rcta. vol20_num2_art:1460

8. CALERO, A.; QUINTERO, E.; OLIVERA, D.; PEÑA K.; PÉREZ, Y. 2019b. Influencia de dos bioestimulantes en el comportamiento agrícola del cultivo del tabaco (Nicotiana tabacum L.). Rev. Fac. Ciencias. (Colombia). 8(1):31-44.

9. CAlERO, A.; QUINTERO, E.; PÉREZ, Y.; OLIVERA, D.; PEÑA, K.; JIMÉNEZ, J. 2019c. Efecto entre microorganismos eficientes y fitomas-e en el incremento agroproductivo del frijol. Biotecnol. Sect. Apropecuario Agroindustrial. (Colombia). 17(1):25-33. https://doi. org/10.18684/bsaa.v17n1.1201

10. CAlero, A.; PÉREZ, Y.; PÉREZ, D. 2016. Efecto de diferentes biopreparados combinado con Fitomas-E en el comportamiento agroproductivo del frijol común (Phaseolus vulgaris L.). Monfragüe Desarro. Resiliente. (España). 7(2):161-176.

11. CALERO, A.; QUINTERO, E.; OLIVERA, D.; PÉREZ, Y.; CASTRO, I.; JIMÉNEZ, J.; LÓPEZ, E. 2018. Respuesta de dos cultivares de frijol común a la aplicación foliar de microorganismos eficientes. Cultivos Tropicales. (Cuba). 39(3):5-10.

12. CALERO, A.; QUINTERO, E.; PÉREZ, Y. 2017. Utilización de diferentes bioproductos en la producción de frijol común (Phaseolus vulgaris L). Agrotec. Cuba. (Cuba). 41(1):1-13.
13. FLEITAS, M.; BENÍTEZ, T.; CASTILLO, R. 2013. Evaluación del humus de lombriz y estiércol bovino en la producción del rábano (Raphanus sativus L.) en condiciones de organopónico. Rev. Pakamuros. 1: 2306-9805.

14. GÓMEZ, L.; RODRÍGUEZ, M.G.; ENRIQUE, R.; MIRANDA, I.; GONZÁLEZ, E. 1986. Factores limitantes de los rendimientos y calidad de las cosechas en la producción protegida de hortalizas en Cuba. Rev. Protección Veg. (Cuba). 24(2):117-122.

15. GUTIÉRREZ, F.A.; GARCÍA, R.C.; RINCÓN, R.; ABUD, M.; OLIVA, M.A.; CRUZ, M.J.; DENDOOVEN, L. 2008. Formulation of a liquid fertilizer for sorghum (Sorghum bicolor (L.) Moench) using vermicompost leachate. Bioresour. Technol. (Holanda). 99(11):6174-6180. http:// dx.doi.org/10.1016/J.BIORTECH.2007.12.043

16. JARECKI, M.K.; CHONG, C.; VORONEY, R.P. 2005. Evaluation of compost leachates for plant growth in hydroponic culture. J. Plant Nutr. (Alemania). 28(4):651667. http://dx.doi.org/10.1081/PLN-200052639

17. KUMAR, C.; SARAF, M. 2015. Plant growth promoting Rhizobacteria (PGPR): a review. J. Agric. Res. Dev. (Nigéria). 5(2):108-119. https://doi.org/10.13140/RG.2.1.5171.2164

18. LIRIANO, R.; NÚÑEZ, D.; HERNÁNDEZ, L.; CASTRO, A. 2015. Evaluación de microorganismos eficientes y Trichoderma harzianum en la producción de posturas de cebolla (Allium cepa L.). Cent. Agrícola. (Cuba). 42(4):25-32.

19. LÓPEZ, E.; CALERO, A.; GÓMEZ, Y.; GIL, Z.; HENDERSON, D.; JIMÉNEZ, J. 2017. Efecto agronómico del biosólido en cultivo de tomate (Solanum lycopersicum): control biológico de Rbizoctonia solani. Cultiv. Trop. (Cuba). 38(1):13-23.

20. LUNA, M.; MESA, J. 2016. Microorganismos eficientes y sus beneficios para los agricultores. Agroecosistemas. (Cuba). 4(2):31-40.

21. MOHAMMADI, A.; OMID, M. 2010. Economical analysis and relation between energy inputs and yield of greenhouse cucumber production in Iran. Appl. Energy. (Estados Unidos). 87(1):191-196. http://dx.doi.org/10.1016/J. APENERGY.2009.07.021

22. NÚÑEZ, D.B.; LIRIANO, R.; PÉREZ, Y.; PLACERES, I.; SIANEH, G. 2017. Respuesta de Dancus carota, L. a la aplicación de microorganismos nativos en condiciones de organopónico. Cent. Agrícola. (Cuba). 44(2):29-35.

23. OLIVERA, D.; AYALA, J.; CALERO, A.; SANTANA, M.; HERNÁNDEZ, A. 2014. Prácticas agroecológicas en la provincia de Sancti Spíritus, Cuba. Microorganismos 
eficientes (EM), una tecnología apropiada sobre bases agroecológicas. Ciência Tecnol. Soc. na Construção da Agroecol. (Brasil). 7(1):77-83.

24. OLIVERA, D.; LEIVA, L.; CALERO, A.; MELÉNDREZ, J.F. 2015. Empleo de microorganismos nativos multipropósitos (MNM) en el comportamiento agro-productivo de cultivos hortícolas. Agrotec. Cuba. (Cuba). 39(7):34-42.

25. PANT, A.P.; RADOVICH, T.J.; HUE, N.V.; TALCOT'T, S.T.; KRENEK, K.A. 2009. Vermicompost extracts influence growth, mineral nutrients, phytonutrients and antioxidant activity in pak choi (Brassica rapa cv. Bonsai, Chinensis group) grown under vermicompost and chemical fertilizer. J. Sci. Food Agric. (Estados Unidos). 89(14):2383-2392. http://dx.doi.org/10.1002/jsfa.3732

26. PEDRAZA, R.O.; TEIXEIRA, K.R.; FERNÁNDEZ, A.; DE SALAMONE, I.G.; BACA, B.E.; AZCÓN, R.; BALDANI, V.L.; BONILLA, R. 2010. Microorganismos que mejoran el crecimiento de las plantas y la calidad de los suelos. Rev. Corpoica Cienc. y Tecnológica Agropecu. (Colombia). 11(2):155-164. http://dx.doi.org/10.21930/rcta.vol11_ num2_art:206

27. PRECIADO, P.; FORTIS, M.; GARCÍA, J.L.; RUEDA, E.O.; ESPARZA, J.R.; LARA, A.; SEGURA, M.A.; OROZCO, J.A. 2011. Evaluación de soluciones nutritivas orgánicas en la producción de tomate en invernadero. Interciencia. (Colombia). 36(9):689-693.

28. PRECIADO, P.; GARCÍA, J.L.; SEGURA, M.Á.; SALAS, L.; AYALA, A.V.; GARAY, A.; ESPARZA, J.R.; RIVERA, E.; TROYO, E. 2014. Efecto del lixiviado -de vermicomposta en la producción hidropónica de maíz forrajero. Terra Latinoam. (México). 32(4):333-338.

29. QUINTERO, E.; CALERO, A.; PÉREZ, Y.; ENRÍQUEZ, L. 2018. Efecto de diferentes bioestimulantes en el rendimiento del frijol común. Cent. Agrícola. (Cuba). 45(3):73-80.

30. RASHID, M.I.; MUJAWAR, L.H.; SHAHZAD, T.; ALMEELBI, T.; ISMAIL, I.M.I.; OVES, M. 2016. Bacteria and fungi can contribute to nutrients bioavailability and aggregate formation in degraded soils. Microbiol. Res. (Holanda). 183:26-41. http://dx.doi.org/10.1016/j.micres.2015.11.007
31. RAVINDRAN, B.; WONG, J.W.; SELVAM A.; SEKARAN, G. 2016. Influence of microbial diversity and plant growth hormones in compost and vermicompost from fermented tannery waste. Bioresour. Technol. (Holanda). 217:200-204. http://dx.doi.org/1016/J.BIOR'TECH.2016.03.032

32. SINGH, J.S.; PANDEY, V.C.; SINGH, D.P. 2011. Efficient soil microorganisms: A new dimension for sustainable agriculture and environmental development. Agric. Ecosyst. Environ. (Holanda). 140(3-4):339-353. http:// dx.doi.org/10.1016/j.agee.2011.01.017

33. SINGH, R.; GUPTA, R.K.; PATIL, R.T.; SHARMA, R.R.; ASREY, R.; KUMAR, A.; JANGRA, K.K. 2010. Sequential foliar application of vermicompost leachates improves marketable fruit yield and quality of strawberry (Fragaria $\times$ ananassa Duch.). Sci. Hortic. (Amsterdam). 124(1):34-39. http://dx.doi.org/10.1016/J.SCIENTA.2009.12.002

34. SIMRANJIT, K.; KANCHAN, A.; PRASANNA, R.; RANJAN, K.; RAMAKRISHNAN, B.; SINGH, A.K.; SHIVAY, Y.S. 2019. Microbial inoculants as plant growth stimulating and soil nutrient availability enhancing options for cucumber under protected cultivation. World J Microbiol Biotechnol (Ukrania), 35(3):35-51. https://doi.org/10.1007/s11274019-2623-z

35. TEJADA, M.; GONZÁLEZ, J.L.; HERNÁNDEZ, M.T.; GARCÍA, C. 2008. Agricultural use of leachates obtained from two different vermicomposting processes. Bioresour. Technol. (Holanda). 99(14):6228-6232. https://doi. org/10.1016/J.BIORTECH.2007.12.031

36. ZAHEDI, H. 2016. Growth-promoting effect of potassiumsolubilizing microorganisms on some crop species. In: Singh, V.; Meena, R.; Verma, J.; Maurya, B. (eds.). Potassium solubilizing microorganisms for sustainable agriculture. Springer, India, 331p.

37. ZHAO, H.T.; LI, T.P.; ZHANG, Y.; HU, J.; BAI, Y.H.; SHAN Y.H.; KE, F. 2017. Effects of vermicompost amendment as a basal fertilizer on soil properties and cucumber yield and quality under continuous cropping conditions in a greenhouse. J. Soils Sediments. (Estados Unidos). 17:27182730. https://doi.org/10.1007/s11368-017-1744-y. 